\title{
Cystic fibrosis transmembrane conductance regulator in follicular fluid and cumulus cells and its relationship with age
}

\author{
YANQIU WANG ${ }^{*}$, HUI WEI* ${ }^{*}$ YAZHONG JI, FEIPING LIU, ZHIJUN SHEN and XUNYI ZHANG \\ Department of Reproductive Medicine, Tongji Hospital, Tongji University School of Medicine, Shanghai 200065, P.R. China
}

Received April 25, 2020; Accepted October 30, 2020

DOI: $10.3892 /$ etm. 2020.9570

\begin{abstract}
Oocyte quality deteriorates with female age and numerous indicators of oocyte quality exist. In the present study, the levels of cystic fibrosis transmembrane conductance regulator (CFTR) in the follicular fluid (FF) and cumulus cells (CCs) of infertile females in 3 different age groups were assessed to determine the relationship between CFTR and female age. The general features of the 3 groups, including age, body mass index, infertility duration, basal hormone levels and the number of retrieved oocytes were compared. The FF CFTR levels of the 3 groups were also compared and multiple age-related indicators of oocyte quality were analyzed, including the estradiol levels on the human chorionic gonadotropin injection day, the morphologically normal oocyte rate and the available or high-quality embryo rate. Immunofluorescence and PCR analyses were performed to examine CFTR expression in CCs around oocytes. The results indicated differences in general features and several indicators of oocyte quality among the 3 groups and significant differences in CFTR. The FF CFTR level was positively correlated with age, which was confirmed by immunofluorescence and PCR. Collectively, these results indicated that CFTR expression in FF and CCs may be associated with oocyte quality based on the age of individuals undergoing the assisted reproduction technique.
\end{abstract}

\section{Introduction}

The developmental potential of transferred embryos is the major contributing factor to the outcomes of in vitro fertilization (IVF) cycles (1), while oocyte quality is an important factor affecting embryo quality (2). Therefore, assessing

Correspondence to: Dr Xunyi Zhang, Department of Reproductive Medicine, Tongji Hospital, Tongji University School of Medicine, 389 Xincun Road, Shanghai 200065, P.R. China

E-mail: zxymeng@aliyun.com

*Contributed equally

Key words: cystic fibrosis transmembrane conductance regulator, follicular fluid, cumulus cells, oocyte quality, age oocyte quality helps clinicians obtain the best oocytes and subsequently improve the outcomes of IVF treatment. Oocyte quality is influenced by patient-related factors, including age, body mass index (BMI) and infertility type, and external factors, such as ovarian stimulation protocols, laboratory procedures and culture conditions, although age is the most important factor (3-5).

Cystic fibrosis transmembrane conductance regulator (CFTR) is a cyclic adenosine monophosphate (cAMP)-dependent chloride channel protein that is closely related to human reproductive function and participates in multiple reproductive events $(6,7)$. CTFR is the only chloride channel in the uterus and controls secretions of the cervix and uterus to regulate a series of reproductive events such as sperm capacitation, sperm migration, oocyte fertilization and embryo implantation $(8,9)$. When targeted to the plasma membrane, CFTR functions as a small conductance $\mathrm{Cl}^{-}$channel activated by protein kinase A-dependent phosphorylation (10).

To explore novel indices for evaluating oocyte quality based on age, such as CFTR, the present study investigated the relationship between CFTR in the follicular fluid (FF) or cumulus cells (CCs) and age of the individuals.

\section{Materials and methods}

Patients. Between December 2017 and September 2018, 80 couples undergoing assisted reproductive technology (ART) treatment at Tongji Hospital of Tongji University (Shanghai, China) were recruited. Couples had male factor infertility and underwent intracytoplasmic sperm injection (ICSI) treatment were enrolled in this study. Individuals with female infertility risk factors, including fallopian tube obstruction, ovarian factor and underwent in vitro fertilization treatment were excluded from this study. The age of the females was between 25 and 42 years, their BMI ranged from 18 to $27 \mathrm{~kg} / \mathrm{m}^{2}$ and the duration of infertility ranged from 0.6 to 10.2 years. The patients were assigned to 3 groups according to female age (group A, $\leq 30$ years; group B, $\geq 31$ and $\leq 35$ years; and group $\mathrm{C}, \geq 36$ years) and underwent controlled ovarian stimulation with the use of a standard gonadotropin-releasing hormone agonist (GnRH-a) long protocol. GnRH-a (0.1 mg/d; Diphereline; Ipsen Pharma Biotech) was administered by intramuscular injection beginning on the 21 st day of the run-in cycle for 14 days. After the menstruation and when estradiol $\left(\mathrm{E}_{2}\right)<50 \mathrm{pg} / \mathrm{ml}$, which indicated pituitary block, 300 IU of recombinant human follitropin 
(GONAL-f; Merck Serono) was administered until $>2$ follicles $\leq 18 \mathrm{~mm}$ in diameter were observed by vaginal ultrasound. Subsequently, human chorionic gonadotropin (HCG; LiZhu Co., Ltd.) was administered at a dose of $6,000 \mathrm{IU}$ at $36 \mathrm{~h}$ prior to ultrasound-guided transvaginal oocyte retrieval.

\section{Sample collection}

Blood serum sampling. Blood samples were obtained from all females during their menstrual cycles and on the day of HCG administration. Blood samples were kept at room temperature for $0.5 \mathrm{~h}$ and centrifuged at $1,000 \mathrm{x}$ g for $10 \mathrm{~min}$ at $26^{\circ} \mathrm{C}$. Serum was isolated and stored at $-80^{\circ} \mathrm{C}$.

FF collection. FF samples were collected from all females during ultrasound-guided transvaginal oocyte retrieval and FF was collected only from the first aspirated follicle with a diameter $>15 \mathrm{~mm}$. Oocyte retrieval needles were not flushed with any culture medium. Once the oocyte-corona-cumulus complex (OCCC) was found, the FF supernatant was preserved after centrifugation at $1,000 \mathrm{xg}$ for $10 \mathrm{~min}$ at $26^{\circ} \mathrm{C}$ and stored at $-80^{\circ} \mathrm{C}$.

CC retrieval.CCs were collected from each of the 3 groups. Only the oocyte that was aspirated without flushing in the first aspiration was used to obtain cumulus cells in each patient. A petri dish containing $100 \mu 1$ hyaluronidase (80 IU/ml; FUJIFILM Irvine Scientific, Inc.) in the middle and $420-\mu 1$ droplets of gamete handling medium (G-MOPS plus; Vitrolife) around the hyaluronidase droplet was prepared. After incubation for $2 \mathrm{~h}$ at $37^{\circ} \mathrm{C}$ in an atmosphere with $6 \% \mathrm{CO}_{2}$ and $5 \% \mathrm{O}_{2}$ in fertilization medium (G-IVF plus; Vitrolife), the OCCC was digested in hyaluronidase for $1 \mathrm{~min}$ to remove ovarian mural granulosa cells (GCs). Further mechanical denudation was performed in enzyme-free MOPS-buffered medium droplets by repeated aspiration through a Pasteur pipette with an inner diameter of $140 \mu \mathrm{m}$. Finally, only the CCs remaining in the MOPS-buffered medium droplets were collected for future study, while the GCs in enzyme drops were discarded. The $80 \mu \mathrm{l}$ of MOPS-buffered medium containing the $\mathrm{CC}$ suspension was divided into two parts. The first $40 \mu \mathrm{l} \mathrm{CC}$ suspension was seeded in a cell culture dish (353001; Falcon; BD Biosciences), covered with $3 \mathrm{ml}$ oil (OVOIL; FUJIFILM Irvine Scientific, Inc.) and cultured at $37^{\circ} \mathrm{C}$ with $6 \% \mathrm{CO}_{2}$ and $5 \% \mathrm{O}_{2}$ for $24 \mathrm{~h}$ for subsequent immunofluorescence. The second 40- $\mu$ l CC suspension was subjected to total RNA extraction for reverse transcription-quantitative (RT-q)PCR analysis.

Morphologically normal metaphase II (MII) oocyte rate calculation. After denudation, oocytes were determined to be MII oocytes when an extruded first polar body (IPB) was evident and then used for ICSI. In addition, based on the methods reported by Ubaldi and Rienzi (11), morphological assessment of the MII oocytes was performed and included the zona pellucida (ZP), perivitelline space (PVS), IPB and cytoplasm. The morphologically normal MII oocytes of the 3 groups were then calculated using the following formula: The number of morphologically normal MII oocytes/the number of total MII oocytes.

ICSI procedure and embryo culture. ICSI was performed on the heated plate $\left(37^{\circ} \mathrm{C}\right)$ of an inverted microscope (IX73; Olympus Corp.) with a micromanipulator system (Narishige).
MII oocytes were selected for ICSI according to the method described in the Textbook of Assisted Reproductive Technologies, Third Edition (12). The injected oocytes were cultured in embryo culture medium (G-1 plus; Vitrolife) and in tissue culture dishes (cat. no. 353001; Falcon; BD Biosciences) with one oocyte per $30 \mu 1$ medium.

Assessment of cleavage-stage embryos. Morphological assessment of cleavage-stage embryos was performed at $72 \mathrm{~h}$ after ICSI. Cleavage-stage embryo grading was based on the criteria of the Society of ART (13).

Serum hormonal measurements and FF CFTR assessment. Serum $\mathrm{E}_{2}, \mathrm{LH}, \mathrm{FSH}$ and P levels were measured by chemiluminescence immunoassay (I2000sr; Abbott Laboratories). FF CFTR levels were determined using an ELISA kit (cat. no. CSB-EL005292HU; Cusabio Technology LLC) according to the manufacturer's protocol. The CFTR concentration was calculated according to a standard curve.

Immunofluorescence. Immunofluorescence staining was performed on the cell culture dishes containing CCs that had been retrieved from OCCC digestion $24 \mathrm{~h}$ earlier. CCs were fixed with 4\% paraformaldehyde (Sinopharm Co., Ltd.), permeabilized with Triton X-100 (Sangon Co., Ltd.), blocked with normal goat serum (Solarbio Co., Ltd.) and then incubated with mouse anti-CFTR primary antibody (1:100 dilution; cat. no. ab2784; Abcam Co., Ltd.) at $4^{\circ} \mathrm{C}$ overnight. Incubation with polyclonal goat anti-mouse immunoglobulin/FITC secondary antibody (1:50 dilution; cat. no. 33207ES60; Yeasen Co. Ltd.) was performed for $30 \mathrm{~min}$ at room temperature. DAPI dihydrochloride (Sangon Co., Ltd.) staining was performed to visualize nuclei. Stained samples were observed with a DM 4000 microscope (Leica Microsystems). Cell immunofluorescence images were analyzed with MATLAB R2013a (Mathworks).

$R T-q P C R$ analysis of CFTR. CCs from each of the 3 groups were subjected to RT-qPCR to measure CFTR levels. Total RNA from CCs in 40- $\mu$ l cell suspensions was extracted using RNA extraction kit (cat. no. 12183020; PureLink ${ }^{\mathrm{TM}}$ RNA Mini Kit; Invitrogen; Thermo Fisher Scientific, Inc.) and subjected to RT according to the protocol provided by the manufacturer of the BeScript One Step RT-PCR Kit (cat. no. CV003; Shanghai Colegen biological technology Co., Ltd.; http://www.colegen. com/products_16/28.html). RT-qPCR for CFTR was performed with Perfect SYBR Green Master Mix (cat. no. V001; Shanghai Colegen Biological Technology Co., Ltd.; http://www.colegen. com/products_16/31.html) and CFTR levels were normalized to the $\beta$-actin endogenous control gene. A 7500 Sequence Detection System (SDS; Applied Biosystems; Thermo Fisher Scientific, Inc.) was used to monitor the reaction procedure. The primers were as follows: CFTR sense, 5'-TGTAAATGGAGGAGGAAA TG-3' and antisense, 5'-GAGAAGATGATAAGTGGGAAA-3'; and $\beta$-actin sense, 5'-CTGGGACGACATGGAGAAAA-3' and antisense, 5'-AAGGAAGGCTGGAAGAGTGC-3'. The PCR conditions were as follows: $5 \mathrm{~min}$ at $95^{\circ} \mathrm{C}$, then 35 cycles of $15 \mathrm{sec}$ at $95^{\circ} \mathrm{C}$ and $15 \mathrm{sec}$ at $60^{\circ} \mathrm{C}$, followed by $10 \mathrm{~min}$ at $72^{\circ} \mathrm{C}$. Raw data were analyzed by ABI Prism 7500 SDS Software (Applied Biosystems; Thermo Fisher Scientific, Inc.) The RT-qPCR results were quantified using the $2^{-\Delta \Delta C q}$ method (14). 
Table I. Basic demographic and laboratory data of the patients.

\begin{tabular}{|c|c|c|c|c|}
\hline Parameter & Group A $(n=32)$ & Group B $(n=29)$ & Group C $(n=19)$ & P-value \\
\hline Age (years) & $25.88 \pm 2.01$ & $32.25 \pm 1.19$ & $37.62 \pm 1.47$ & $<0.001$ \\
\hline BMI $\left(\mathrm{kg} / \mathrm{m}^{2}\right)$ & $20.9 \pm 2.1$ & $21.7 \pm 2.2$ & $23.0 \pm 4.8$ & 0.468 \\
\hline Duration of infertility (years) & $3.6 \pm 1.9$ & $2.8 \pm 1.9$ & $7.1 \pm 4.4$ & 0.002 \\
\hline $\mathrm{E}_{2}(\mathrm{pmol} / \mathrm{l})$ & $279.58 \pm 71.02$ & $214 \pm 121.21$ & $195.08 \pm 57.26$ & 0.009 \\
\hline LH (IU/l) & $5.39 \pm 3.19$ & $3.95 \pm 1.58$ & $5.13 \pm 3.27$ & 0.400 \\
\hline FSH (IU/l) & $4.19 \pm 1.4$ & $5.81 \pm 1.59$ & $6.73 \pm 1.6$ & 0.004 \\
\hline $\mathrm{P}(\mathrm{nmol} / \mathrm{l})$ & $1.76 \pm 0.49$ & $1.79 \pm 0.64$ & $1.35 \pm 0.41$ & 0.042 \\
\hline OCCC number & $10.8 \pm 7.2$ & $8.1 \pm 5.0$ & $4.2 \pm 4.5$ & 0.001 \\
\hline
\end{tabular}

Values are presented as the mean \pm standard error of the mean. Groups: A, $\leq 30$ years; $\mathrm{B}, \geq 31$ and $\leq 35$ years; and $\mathrm{C}, \geq 36$ years. BMI, body mass index; $\mathrm{E}_{2}$, estradiol; $\mathrm{LH}$, luteinizing hormone; FSH, follicle-stimulating hormone; P, progesterone; OCCC, oocyte-corona-cumulus complex.

Table II. Indicators of oocyte quality.

\begin{tabular}{|c|c|c|c|c|}
\hline Parameter & Group A $(n=32)$ & Group B $(n=29)$ & Group C $(n=19)$ & P-value \\
\hline CFTR in FF (ng/ml) & $52.94 \pm 25.51$ & $75.29 \pm 29.29$ & $92.68 \pm 29.94$ & $<0.001$ \\
\hline $\mathrm{E}_{2}$ on $\mathrm{HCG}$ injection day (nmol/l) & $10.70 \pm 6.84$ & $10.65 \pm 8.07$ & $5.81 \pm 5.18$ & 0.021 \\
\hline Morphologically normal MII oocyte rate (\%) & 80.34 & 78.91 & 73.56 & 0.066 \\
\hline Available embryo rate $(\%)$ & 63.69 & 80.43 & 60.61 & 0.089 \\
\hline High-quality embryo rate (\%) & 58.33 & 40.58 & 33.33 & 0.019 \\
\hline
\end{tabular}

Values are presented as the mean \pm standard error of the mean or percentage. Groups: A, $\leq 30$ years; B, $\geq 31$ and $\leq 35$ years; and C, $\geq 36$ years $\mathrm{E}_{2}$, estradiol; CFTR, cystic fibrosis transmembrane conductance regulator; FF, follicular fluid; MII, metaphase II; HCG, human chorionic gonadotropin.

Statistical analysis. SPSS 20.0 statistical software (IBM Corp.) was used for data analysis. The measurement data are expressed as the mean \pm standard error of the mean. Statistical analysis for each variable was performed using one-way ANOVA followed by Duncan's post-hoc test. The multiple imputation method was used to manage inconsistent numbers of cases between groups (15). The correlation between CFTR level in $\mathrm{FF}$ and $\mathrm{E}_{2}$ on $\mathrm{HCG}$ injection day was analyzed by Pearson's linear correlation analysis. $\mathrm{P}<0.05$ was considered to indicate statistical significance.

\section{Results}

Comparison of the general conditions of the patients. Basal hormone levels during the menstrual cycles of the 80 female patients in the 3 groups were measured and data on the numbers of OCCCs retrieved were also recorded. No significant differences in BMI and LH were observed among the three groups $(\mathrm{P}>0.05)$. Significant differences in infertility duration, $\mathrm{E}_{2}$, $\mathrm{FSH}, \mathrm{P}$ and $\mathrm{OCCC}$ number were identified among the groups $(\mathrm{P}<0.05$; Table I).

Association of FF CFTR levels with indicators of oocyte quality. The level of FF CFTR on the day of oocyte retrieval in group $\mathrm{C}$ was significantly higher than that in group $\mathrm{A}$ or group B and it was higher in group B than that in group A

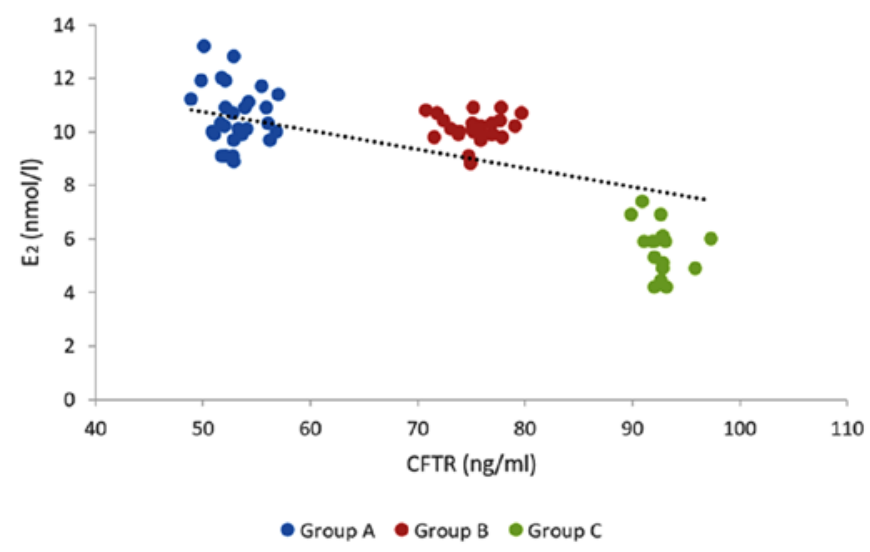

Figure 1. Dot plot of the correlation between CFTR and estradiol levels among the three groups. CFTR, cystic fibrosis transmembrane conductance regulator; $\mathrm{E}_{2}$, estradiol.

$(\mathrm{P}<0.001$; Table II). The level of E2 on HCG injection day decreased with increasing age, where the difference of E2 among the three groups was statistically significant with that in group $\mathrm{C}$ the lowest $(\mathrm{P}<0.05$; Table II). A significant but weak negative correlation was identified among the three groups of FF CFTR levels and $\mathrm{E}_{2}$ levels on the HCG injection day ( $\mathrm{r}=-0.131 ; \mathrm{P}=0.021$; Fig. 1$)$. There were no statistical 

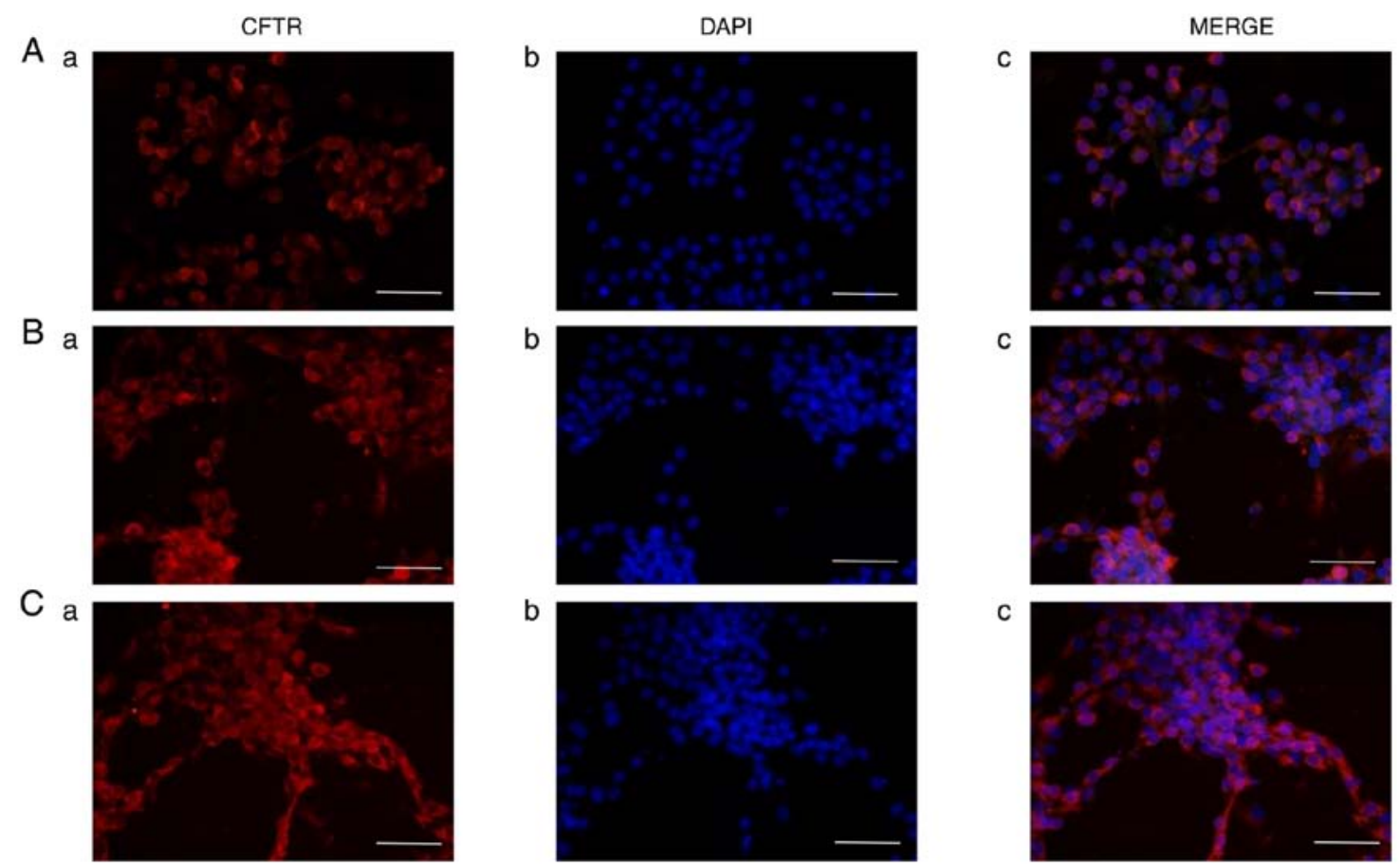

C
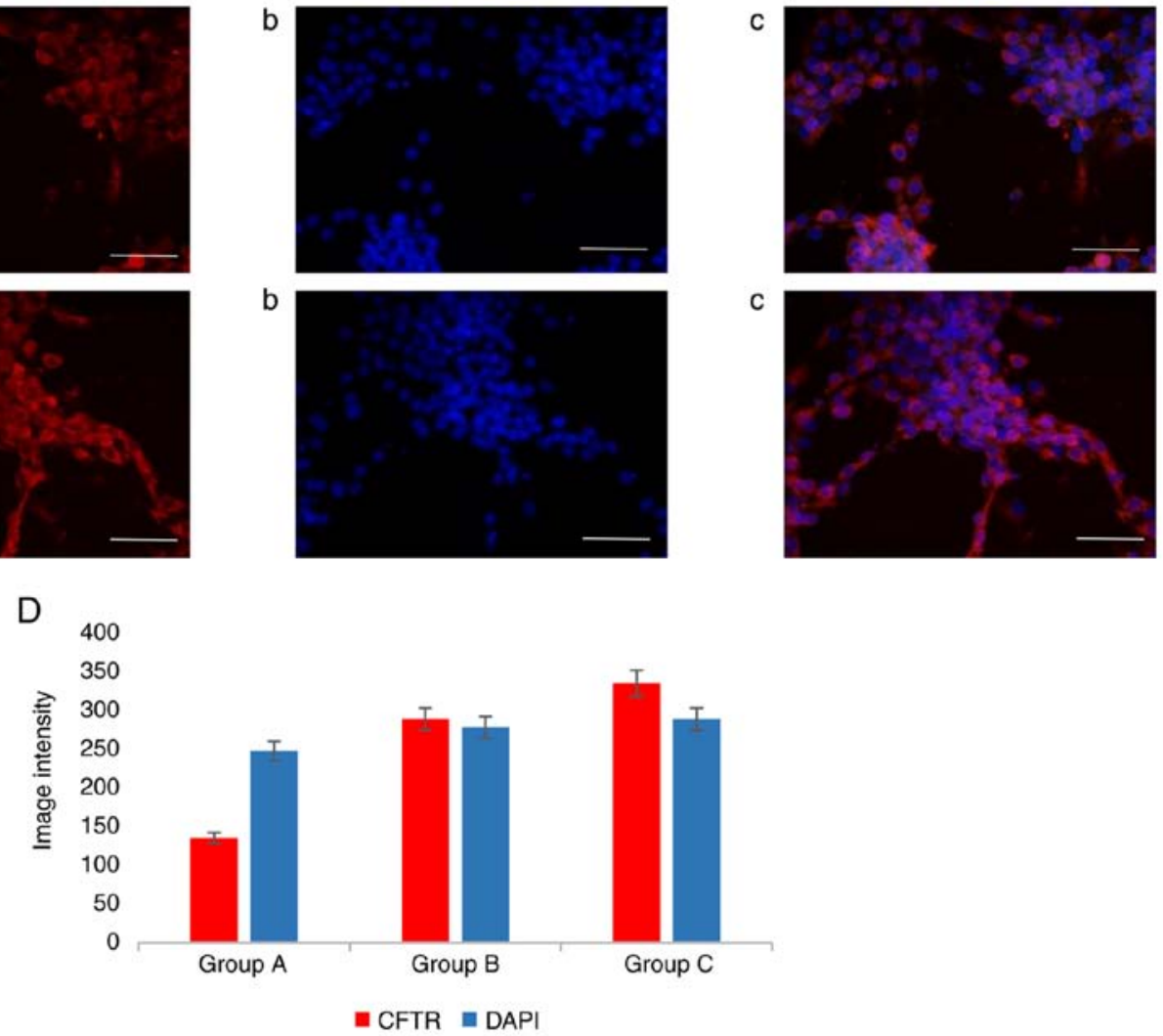

Figure 2. Immunofluorescence staining of CCs for CFTR in (A) group A, (B) group B and (C) group C. On the cell culture dish with CCs, CFTR (red) was readily detected in the cytoplasm. The nuclei were stained with DAPI (blue) (magnification, $\mathrm{x} 400$; scale bar, $50 \mu \mathrm{m}$ ). (D) Fluorescence intensity histogram for the 3 groups. Groups: A, $\leq 30$ years; B, $\geq 31$ and $\leq 35$ years; and C, $\geq 36$ years. CCs, cumulus cells; CFTR, cystic fibrosis transmembrane conductance regulator.

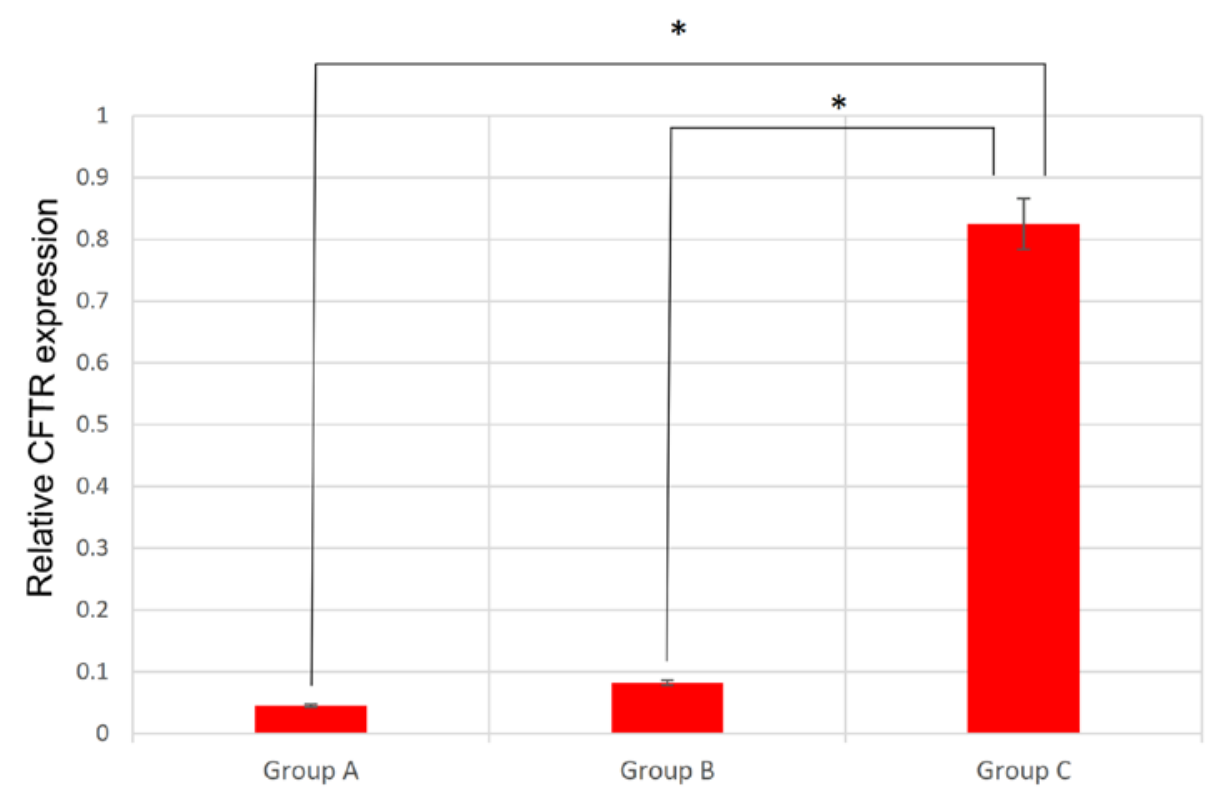

Figure 3. Reverse transcription-quantitative PCR analysis of CFTR and $\beta$-actin expression. ${ }^{*} \mathrm{P}<0.01$. Groups: $\mathrm{A}, \leq 30$ years; $\mathrm{B}, \geq 31$ and $\leq 35$ years; and $\mathrm{C}$, $\geq 36$ years. CFTR, cystic fibrosis transmembrane conductance regulator. 
differences in the morphologically normal MII oocyte rate and available embryo rate among the three groups, but the rate of high-quality embryos declined with age, with that in group $\mathrm{C}$ the lowest $(\mathrm{P}<0.05$; Table II).

Localization and expression of CFTR in cumulus GCs. CFTR expression in CCs (red expression) was detected by immunofluorescence staining and was consistent with that denoted in Gene Cards ${ }^{\circledR}$. The fluorescence intensity of CFTR in group $\mathrm{C}$ was higher than that in group $\mathrm{B}$, while group $\mathrm{A}$ had the lowest intensity (Fig. 2).

RT-qPCR analysis of CFTR. There are $~ 500 \mathrm{CCs}$ around each oocyte and half of the total CCs in each group were used for RT-qPCR. Due to the limitation of sample size analysis in each group was performed twice. The RT-qPCR results were analyzed using the $2^{-\Delta \Delta \mathrm{Cq}}$ method. Analysis of the three groups suggested that the mean $2^{-\Delta \Delta \mathrm{Cq}}$ value of two experimental replicates increased with age and there were significant differences between groups $\mathrm{A}$ and $\mathrm{C}$, and between groups $\mathrm{B}$ and $\mathrm{C}$ $(\mathrm{P}<0.01)$, where group $\mathrm{C}$ had the highest value of $2^{-\Delta \Delta C q}$ (Fig. 3).

\section{Discussion}

Among the patient groups of the present study, differences in proven age-associated indicators were identified, suggesting that the selected patients were representative of the general population (16). Numerous indicators of oocyte quality exist, but each method has its own shortcomings (17). Oocyte morphological assessment is the most widely used method in clinical practice; a normal human MII oocyte should have a round and clear ZP, a small PVS containing a single IPB without fragmentation and pale, moderately granular cytoplasm with no inclusions (18). However, oocyte morphological assessment may be performed only after oocyte denudation in ICSI cases and potential mechanisms that change the appearance of the oocyte are multifactorial (19). Age and the $\mathrm{E}_{2}$ level on the HCG day are indirect indicators (20). Embryo quality reflects oocyte quality only retrospectively and neglects sperm-related factors (21). Therefore, an index that is able to evaluate oocyte quality prior to IVF is still required.

The present results indicated that with increasing age, no significant changes in certain commonly used indicators of oocyte quality occurred, although CFTR was more positively correlated with age than these indicators. The CFTR is an anion channel regulated by CAMP-dependent phosphorylation and is expressed in epithelial cells of a wide variety of tissue types, including the reproductive tracts (22). CFTR affects factors including sperm transport, blastocyst implantation, sperm capacitation and function by regulating fluid volume and bicarbonate secretion in the reproductive tract (23).

The cumulus GC layer regulates oocyte maturation and development through complex mechanisms such as gap junctions (23). Detecting gene expression in GCs may reflect changes in the follicular microenvironment and further provide relevant information on oocyte development (24). Evaluating egg quality and embryonic development potential indirectly is helpful. In the present study, CFTR expression in CCs was detected by indirect immunofluorescence and the results suggested that CFTR was mainly localized to the cumulus
GC cytoplasm and membrane. The RT-qPCR results also further demonstrated that CFTR gene expression in cumulus GCs may have a positive correlation with age and may affect the outcome of embryo development. In order to clarify the relationship between the mature oocytes and the patient's age, only the FF and CCs around mature oocytes obtained by the first aspiration without rinsing were collected. FF or CCs were excluded if there was no OCCC found or the oocytes were immature. Therefore, the number of cases enrolled is limited, particularly in group $\mathrm{C}$. To verify this result, it is required to increase the sample sizes of the different age groups.

In assisted reproductive technology, FF metabonomics may be combined with other clinical factors such as age to predict the quality of oocytes and other important information (25). Multiple studies have examined correlations between lipid metabolism, amino acid metabolism or glucose metabolism in FF and oocyte quality (26). Certain studies have indicated that the levels of fatty acids in FF are related to oocyte maturity and that certain changes in fatty acids may improve oocyte quality (27). Regarding amino acid metabolism, amino acids in FF have an important role in oocyte maturation and fertilization and evaluating oocyte quality according to amino acid levels in FF is a current research hotspot (28). Regarding glycometabolism, Robker et al (29) observed that glucose levels in the FF of fertilized eggs with failed cleavage are significantly higher compared with those in the FF of fertilized eggs with normal cleavage, indicating that low oocyte quality is related to high glucose levels in FF. In the present study, CFTR levels were compared with several classical indexes used to evaluate oocyte quality to identify novel correlations and the correlation between age and the CFTR level in FF was confirmed for the first time, to the best of our knowledge, i.e. that the CFTR level in FF is positively correlated with age. Due to the limited cases enrolled in this study, further research is needed. Although the identification of specific predictive biochemical markers is not yet possible, this method has potential application value. FF metabonomics analysis is expected to open a new chapter in the screening of high-quality oocytes and embryos.

\section{Acknowledgements}

Not applicable.

\section{Funding}

The present study was supported by grants from the Tongji Hospital Clinical Research Project [grant nos. ITJ (QN) 1809 and ITJ (QN) 1813] and the Shanghai Key Laboratory of Embryo Original Diseases (grant no. Shelab201903).

\section{Availability of data and materials}

The datasets used and/or analyzed during the current study are available from the corresponding author on reasonable request.

\section{Authors' contributions}

YW and HW performed the experiments. HW, FL, YJ and ZS analyzed the data. XZ and YW conceived the study and 
wrote the manuscript. All authors read and approved the final manuscript.

\section{Ethics approval and consent to participate}

The present study was approved by the Ethics Committee of Shanghai Tongji Hospital (Shanghai, China). Written informed consent was provided by each patient prior to inclusion in this study.

\section{Patient consent for publication}

Not applicable.

\section{Competing interests}

The authors declare that they have no competing interests.

\section{References}

1. Moragianni D, Dryllis G, Andromidas P, Kapeta-Korkouli R, Kouskouni E, Pessach I, Papalexis P, Kodonaki A, Athanasiou N, Pouliakis A and Baka S: Genital tract infection and associated factors affect the reproductive outcome in fertile females and females undergoing in vitro fertilization. Biomed Rep 10: 231-237, 2019.

2. Wang X, Cai J, Liu L, Jiang X, Li P, Sha A and Ren J: Association between outdoor air pollution during in vitro culture and the outcomes of frozen-thawed embryo transfer. Hum Reprod 34 441-451, 2019.

3. Smits MAJ, Wong KM, Mantikou E, Korver CM, Jongejan A, Breit TM, Goddijn M, Mastenbroek S and Repping S: Age-related gene expression profiles of immature human oocytes. Mol Hum Reprod 24: 469-477, 2018.

4. Aboulmakarim S, Benbella A, Hardizi H and Bezad R: Retrospective assessment of an assisted reproductive technology method. Ann Biol Clin (Paris) 76: 11-21, 2018.

5. Colaco S and Sakkas D: Paternal factors contributing to embryo quality. J Assist Reprod Genet 35: 1953-1968, 2018.

6. Chan LN, Tsang LL, Rowlands DK, Rochelle LG, Boucher RC, Liu CQ and Chan HC: Distribution and regulation of $\mathrm{ENaC}$ subunit and CFTR mRNA expression in murine female reproductive tract. J Membr Biol 185: 165-176, 2002.

7. Su M, Guo Y, Zhao Y, Korteweg C and Gu J: Expression of cystic fibrosis transmembrane conductance regulator in paracervical ganglia. Biochem Cell Biol 88: 747-55, 2010.

8. Zheng XY, Chen GA and Wang HY: Expression of cystic fibrosis transmembrane conductance regulator in human endometrium. Hum Reprod 19: 2933-2941, 2004.

9. Field PD and Martin NJ: CFTR mutation screening in an assisted reproductive clinic. Aust N Z J Obstet Gynaecol 51: 536-539, 2011.

10. Sheppard DN and Welsh MJ: Structure and function of the CFTR chloride channel. Physiol Rev 79 (Suppl 1): S23-S45, 1999.

11. Ubaldi F and Rienzi L: Morphological selection of gametes. Placenta 29 (Suppl B): S115-S120, 2008.

12. David KG, Ariel W, Colin MH and Zeev S: Textbook of Assisted Reproductive Technologies. 3rd edition. Informa Healthcare Corp., pp171-180, 2009.

13. Racowsky C, Vernon M, Mayer J, Ball GD, Behr B, Pomeroy KO, Wininger D, Gibbons W, Conaghan J and Stern JE: Standardization of grading embryo morphology. Fertil Steril 94: $1152-1153,2010$
14. Schmittgen TD and Livak KJ: Analyzing real-time PCR data by the comparative C(T) method. Nat Protoc 3: 1101-1108, 2008.

15. Kmetic A, Joseph L, Berger C and Tenenhouse A: Multiple imputation to account for missing data in a survey: Estimating the prevalence of osteoporosis. Epidemiology 13: 437-444, 2002

16. Tarin JJ, Ten J, Vendrell FJ and Cano A: Dithiothreitol prevents age-associated decrease in oocyte/conceptus viability in vitro. Hum Reprod 13: 381-386, 1998.

17. Gosden LV: Oocyte retrieval and quality evaluation. Methods Mol Biol 1154: 343-360, 2014.

18. Camargos M, Rodrigues JK, Lobach VN, El Cury-Silva T, Nunes MEG, Camargos AF and Reis FM: Human oocyte morphometry before and after cryopreservation: A prospective cohort study. Cryobiology 88: 81-86, 2019.

19. Hajiyavand AM, Saadat M, Abena A, Sadak F and Sun X: Effect of injection speed on oocyte deformation in ICSI. Micromachines (Basel) 10: 226, 2019.

20. Chen H, Zheng JB, Wang DM, Xing $\mathrm{H}$ and Wang $\mathrm{H}$ : Association between vascular endothelial growth factor and clinical outcomes of IVF-ET/ICSI. J Coll Physicians Surg Pak 29: 19-23, 2019.

21. Devreker F, Pogonici E, De Maertelaer V, Revelard P, Van Den Bergh M and Englert Y: Selection of good embryos for transfer depends on embryo cohort size: Implications for the 'mild ovarian stimulation' debate. Hum Reprod 14: 3002-3008, 1999.

22. Chan HC, Ruan YC, He Q, Chen MH, Chen H, Xu WM, Chen WY, Xie C, Zhang XH and Zhou Z: The cystic fibrosis transmembrane conductance regulator in reproductive health and disease. J Physiol 587: 2187-2195, 2009.

23. Dehghan Z, Mohammadi-Yeganeh S and Salehi M: MiRNA-155 regulates cumulus cells function, oocyte maturation, and blastocyst formation. Biol Reprod 103: 548-559, 2020.

24. Bonnet A, Cabau C, Bouchez O, Sarry J, Marsaud N, Foissac S, Woloszyn F, Mulsant P and Mandon-Pepin B: An overview of gene expression dynamics during early ovarian folliculogenesis: Specificity of follicular compartments and bi-directional dialog. BMC Genomics 14: 904, 2013.

25. Bracewell-Milnes T, Saso S, Abdalla H, Nikolau D, Nornam-Taylor J, Johnson M, Holmes E and Thum MY: Metabolomics as a tool to identify biomarkers to predict and improve outcomes in reproductive medicine: A systematic review. Hum Reprod Update 23: 723-736, 2017.

26. Arya BK, Haq AU and Chaudhury K: Oocyte quality reflected by follicular fluid analysis in poly cystic ovary syndrome (PCOS): A hypothesis based on intermediates of energy metabolism. Med Hypotheses 78: 475-478, 2012.

27. Fayezi S, Leroy J, Ghaffari NM and Darabi M: Oleic acid in the modulation of oocyte and preimplantation embryo development. Zygote 26: 1-13, 2018.

28. Anchordoquy JP, Lizarraga RM, Anchordoquy JM, Nikoloff N, Rosa DE, Fabra MC, Peral-Garcia P and Furnus CC: Effect of cysteine, glutamate and glycine supplementation to in vitro fertilization medium during bovine early embryo development. Reprod Biol 19: 349-355, 2019.

29. Robker RL, Akison LK, Bennett BD, Thrupp PN, Chura LR, Russell DL, Lane $M$ and Norman RJ: Obese women exhibit differences in ovarian metabolites, hormones, and gene expression compared with moderate-weight women. J Clin Endocrinol Metab 94: 1533-1540, 2009.

This work is licensed under a Creative Commons Attribution-NonCommercial-NoDerivatives 4.0 International (CC BY-NC-ND 4.0) License. 